\title{
Review
}

Alexander A. Auer*, Sébastien Cap, Markus Antonietti, Serhiy Cherevko, Xiaohui Deng, Georgios Papakonstantinou, Kai Sundmacher, Sebastian Brüller, Iryna Antonyshyn, Nikolaos Dimitratos, Robert J. Davis, Karl-Heinz Böhm, Nina Fechler, Simon Freakley, Yuri Grin, Brent T. Gunnoe, Hossein Haj-Hariri, Graham Hutchings, Haiwei Liang, Karl J. J. Mayrhofer, Klaus Müllen, Frank Neese, Chinmoy Ranjan, Meenakshisundaram Sankar, Robert Schlögl, Ferdi Schüth, loannis Spanos, Martin Stratmann, Harun Tüysüz, Tanja Vidakovic-Koch, Youngmi Yi and Giovanni Zangari

\section{MAXNET Energy - Focusing Research in Chemical Energy Conversion on the Electrocatlytic Oxygen Evolution}

DOI 10.1515/green-2015-0021

Received November 16, 2015; accepted December 11, 2015

*Corresponding author: Alexander A. Auer, Max Planck Institute for Chemical Energy Conversion, Stiftstr 34-36, 45470 Mülheim an der Ruhr, Germany, E-mail: Alexander.Auer@cec.mpg.de

Sébastien Cap, Fritz Haber Institute of the Max Planck Society, Faradayweg 4-6, 14195 Berlin, E-mail: sebastiencap@fhi-berlin.mpg.de Markus Antonietti, Department of Colloid Chemistry, Max Planck Institute of Colloids and Interfaces, MPI Campus Golm, Am Mühlenberg 1, 14476 Potsdam, Germany

Serhiy Cherevko, Max-Planck-Institut für Eisenforschung, Max-Planck-Straße 1, 40237 Düsseldorf, Germany

Xiaohui Deng, Max-Planck-Institut für Kohlenforschung, Kaiser-Wilhelm-Platz 1, D-45470 Mülheim an der Ruhr, Germany

Georgios Papakonstantinou, Max Planck Institute for Dynamics of Complex Technical Systems, Sandtorstraße 1, D-39106 Magdeburg, Germany Kai Sundmacher, Max Planck Institute for Dynamics of Complex Technical Systems, Sandtorstraße 1, D-39106 Magdeburg, Germany; Otto-vonGuericke University Magdeburg, Process Systems Engineering, Universitätsplatz 2, D-39106 Magdeburg, Germany

Sebastian Brüller, Max Planck Institute for Polymer Research, Ackermannweg 10, 55128 Mainz, Germany, E-mail: brueller@mpip-mainz.mpg.de Iryna Antonyshyn, Max Planck Institute for Chemical Physics and Solids, Nöthnitzer Str. 40, 01187 Dresden, Germany,

E-mail: Iryna.Antonyshyn@cpfs.mpg.de

Nikolaos Dimitratos, Cardiff University, Cardiff, CF10 3XQ, UK

Robert J. Davis, University of Virginia, Charlottesville, VA, USA

Karl-Heinz Böhm, Max Planck Institute for Chemical Energy Conversion, Stiftstr 34-36, 45470 Mülheim an der Ruhr, Germany

Nina Fechler, Department of Colloid Chemistry, Max Planck Institute of Colloids and Interfaces, MPI Campus Golm, Am Mühlenberg 1, 14476

Potsdam, Germany, E-mail: Nina.Fechler@mpikg.mpg.de

Simon Freakley, Cardiff University, Cardiff, CF10 3XQ, UK, E-mail: freakleys@cardiff.ac.uk

Yuri Grin, Max Planck Institute for Chemical Physics and Solids, Nöthnitzer Str. 40, 01187 Dresden, Germany

Brent T. Gunnoe: E-mail: tbg7h@eservices.virginia.edu, Hossein Haj-Hariri, University of Virginia, Charlottesville, VA, USA

Graham Hutchings, Cardiff University, Cardiff, CF10 3XQ, UK

Haiwei Liang, Max Planck Institute for Polymer Research, Ackermannweg 10, 55128 Mainz, Germany

Karl J. J. Mayrhofer, Max-Planck-Institut für Eisenforschung, Max-Planck-Straße 1, 40237 Düsseldorf, Germany, E-mail: mayrhofer@mpie.de Klaus Müllen, Max Planck Institute for Polymer Research, Ackermannweg 10, 55128 Mainz, Germany

Frank Neese, Chinmoy Ranjan, Max Planck Institute for Chemical Energy Conversion, Stiftstr 34-36, 45470 Mülheim an der Ruhr, Germany Meenakshisundaram Sankar, Cardiff University, Cardiff, CF10 3XQ, UK, E-mail: sundmacher@mpi-magdeburg.mpg.de

Robert Schlögl, Max Planck Institute for Chemical Energy Conversion, Stiftstr 34-36, 45470 Mülheim an der Ruhr, Germany; Fritz Haber Institute of the Max Planck Society, Faradayweg 4-6, 14195 Berlin

Ferdi Schüth, Max-Planck-Institut für Kohlenforschung, Kaiser-Wilhelm-Platz 1, D-45470 Mülheim an der Ruhr, Germany Ioannis Spanos, Max Planck Institute for Chemical Energy Conversion, Stiftstr 34-36, 45470 Mülheim an der Ruhr, Germany Martin Stratmann, Max-Planck-Institut für Eisenforschung, Max-Planck-Straße 1, 40237 Düsseldorf, Germany

Harun Tüysüz, Max-Planck-Institut für Kohlenforschung, Kaiser-Wilhelm-Platz 1, D-45470 Mülheim an der Ruhr, Germany,

E-mail: tueysuez@kofo.mpg.de

Tanja Vidakovic-Koch, Max Planck Institute for Dynamics of Complex Technical Systems, Sandtorstraße 1, D-39106 Magdeburg, Germany Youngmi Yi, Max Planck Institute for Chemical Energy Conversion, Stiftstr 34-36, 45470 Mülheim an der Ruhr, Germany

Giovanni Zangari, University of Virginia, Charlottesville, VA, USA 
Abstract: MAXNET Energy is an initiative of the Max Planck society in which eight Max Planck institutes and two external partner institutions form a research consortium aiming at a deeper understanding of the electrocatalytic conversion of small molecules. We give an overview of the activities within the MAXNET Energy research consortium. The main focus of research is the electrocatalytic water splitting reaction with an emphasis on the anodic oxygen evolution reaction (OER). Activities span a broad range from creation of novel catalysts by means of chemical or material synthesis, characterization and analysis applying innovative electrochemical techniques, atomistic simulations of state-ofthe-art x-ray spectroscopy up to model-based systems analysis of coupled reaction and transport mechanisms. Synergy between the partners in the consortium is generated by two modes of cooperation - one in which instrumentation, techniques and expertise are shared, and one in which common standard materials and test protocols are used jointly for optimal comparability of results and to direct further development. We outline the special structure of the research consortium, give an overview of its members and their expertise and review recent scientific achievements in materials science as well as chemical and physical analysis and techniques. Due to the extreme conditions a catalyst has to endure in the OER, a central requirement for a good oxygen evolution catalyst is not only its activity, but even more so its high stability. Hence, besides detailed degradation studies, a central feature of MAXNET Energy is a standardized test setup/protocol for catalyst stability, which we propose in this contribution.

Keywords: electrocatalysis, chemical energy conversion, oxygen evolution

\section{Introduction}

The transition to an economy in which the sun will be the primary energy source, either directly - for example using photovoltaics - or indirectly - like using hydro- or wind power - will require finding solutions for fundamental scientific and technological problems. Undoubtedly, one of the key processes will be storing abundant but fluctuating energy for example in order to sustain industry branches with continuous energy demand or to power mobile applications [1]. One possible scenario for a storage solution would be to use abundant and readily available compounds, such as water or carbon dioxide that can be converted chemically into energy carriers like hydrogen, alkanes or alcohols. Advantages of energy carriers like methanol would be high energy density as well as an already available infrastructure for liquid fuels [2].
While the idea to use energy from renewable sources to split water into hydrogen [3] or to capture carbon dioxide and convert it to methanol as chemical storage medium (fuel) is debatable as the technological solution [4], it provides an illustrative example demonstrating which key technologies are required - irrespective of which processes, carriers or systems will be realized in detail. The common motif will be the chemical conversion of small molecules like water, oxygen, hydrogen and carbon dioxide (not to forget nitrogen and ammonia), which heavily relies on potent catalysts to shift uphill chemical reactions into technically manageable regimes. While the final practicability and efficiency of such a reaction system will mainly rely on technical solutions like the design and optimization of devices, the driving force for innovation will be insight gained from scientific investigations on fundamental processes of catalysis and electrocatalysis.

However, especially in the field of electrocatalysis a knowledge-driven development of novel technologies is very difficult, as processes at electrified multi-phase interfaces are among the most formidable and complex phenomena in chemistry and physics. Compared to, for example, heterogeneous catalysis at a solid-gas interface like in the Haber-Bosch process, electrocatalysis contains two more dimensions of complexity - the presence of a solvent and the presence of the applied electrochemical potential. Regarding processes like oxygen or hydrogen evolution in water splitting electrocatalysis, gas-liquid-solid three phase boundaries play an essential role. In the oxygen evolution reaction (OER), four successive electron/proton transfer steps occur at the catalyst surface. The catalyst itself is subject to phase transitions that occur under electrochemical potential or upon adsorption of molecular species. Furthermore, the omnipresent solvent - water - itself is a reactant. At small length scales, surface structures have to be rationalized by means of complex phase diagrams, which are functions of composition of the surface and electrolyte, $\mathrm{pH}$ and the electrochemical potential. Possible reactions are subdivided into outer sphere and inner sphere, of which the latter are the more complex due to the involvement of adsorbed species, but which are naturally also more relevant for electrocatalysis. At larger length scales, considering diffusion is an essential prerequisite for understanding the basics of electrochemistry. This does not only concern the diffusion of reactants to the active site or the diffusion of charge carriers in solution but also processes like proton transport in the membrane electrode assembly.

Last but not least - at long timescales - many of the components in devices like fuel cells or electrolysers suffer from degradation due to the very harsh conditions in processes, such as the ORR and OER. For example, if an 


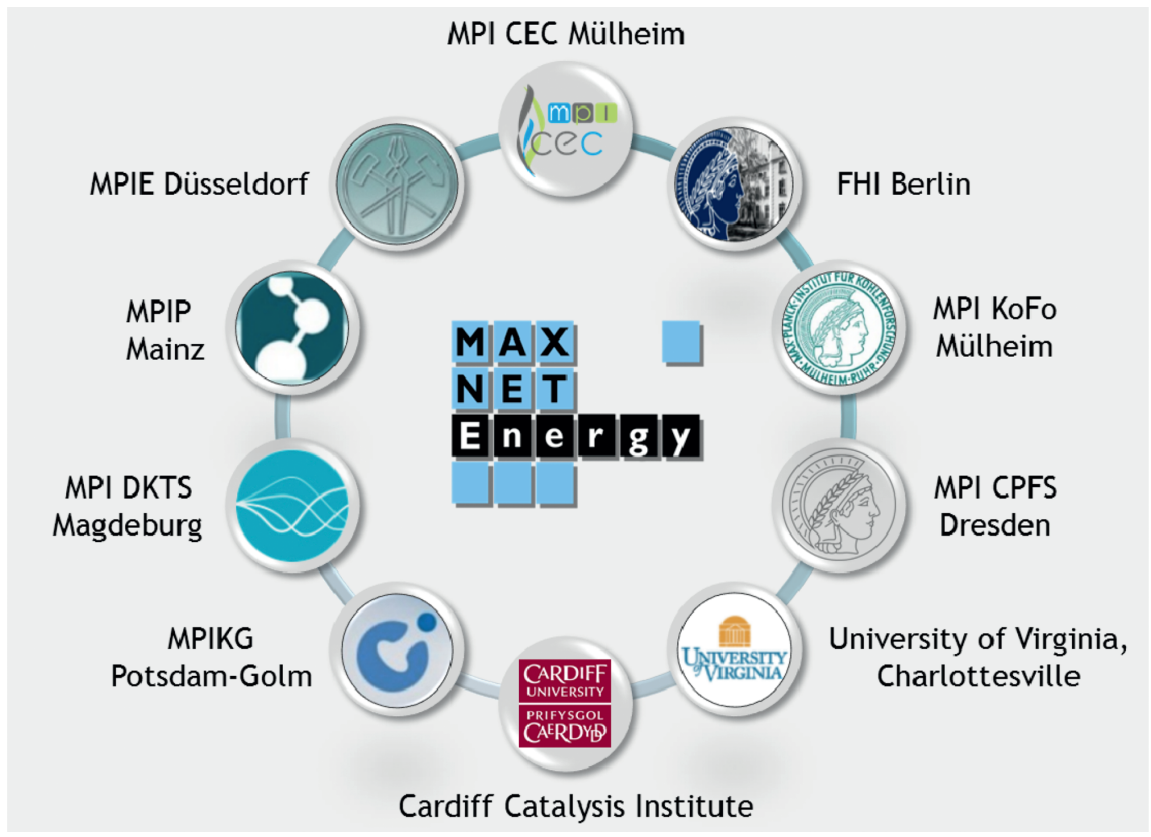

Figure 1: Participants of the MAXNET Energy consortium: Max Planck Institute for Chemical Energy Conversion (MPI CEC), Fritz Haber Institute (FHI), Max Planck Institute for Coal Research (MPI KoFo), Max Planck Institute for Chemical Physics of Solids (MPI CPFS), University of Virginia, Cardiff Catalysis Institute (CCI), Max Planck Institute for Colloids and Interfaces (MPIKG), Max Planck Institute for the Dynamics of Technical Systems(MPI DKTS), Max Planck Institute for Polymer Research (MPIP) and Max-Planck-Institut für Eisenforschung (MPIE).

electrode on which oxygen is evolved operates around $2 \mathrm{~V}$ vs SHE, which is common for typical applications, this corresponds to the chemical oxidation power of chemical oxidants like permanganate, hydrogen peroxide or ozone Figure 1.

\subsection{The MAXNET energy initiative of the Max Planck Society}

The diversity and nature of the challenges described above has been an attractive playground for many groups in the scientific community in general and within the Max Planck Society specifically. Besides the departments of internationally acknowledged capacities with a focus on electrochemistry like Martin Stratmann, the current president of the Max Planck Society, many activities focus on the electrocatalytic activation of small molecules.

Institutes with background in chemical engineering have become interested in electrolyser systems, institutes that focus on catalytic processes have developed activities in electrocatalysis, groups with expertise in materials science work on novel catalyst or membrane materials and groups working in the field of theoretical chemistry have been interested in exploring elementary processes on electrified interfaces. This is why the Max Planck Society has made a commitment to support the many efforts on catalysis in energy conversion in a large research consortium focused in synergy between the institutes (see Figure 1). The MAXNET Energy initiative funds eight Max Planck Institutes all over Germany to employ motivated young scientists that work on energy conversion related topics with the function to form a network of focused cooperation.

The MAXNET Energy originated from the ENERCHEM initiative of the Max Planck Society that was founded in 2004 and has successfully brought together scientists within the MPG from different fields of chemistry, physics and engineering until 2011. After the decision to renew the very successful energy conversion initiative as MAXNET Energy, the kickoff meeting was held in December 2013 (Figure 2). Since then, quarterly meetings have been held at the newly entitled Max Planck Institute for Chemical Energy Conversion in Mülheim an der Ruhr and the Harnack Haus in Berlin. The first focused workshop on catalyst stability was held in November 2015. With the inclusion of two external members in 2014, the MAXNET Energy consortium reached its full size and potential. As external members, the Cardiff Catalysis Institute (UK) and the University of Virginia (USA) contribute essential expertise and complete the 


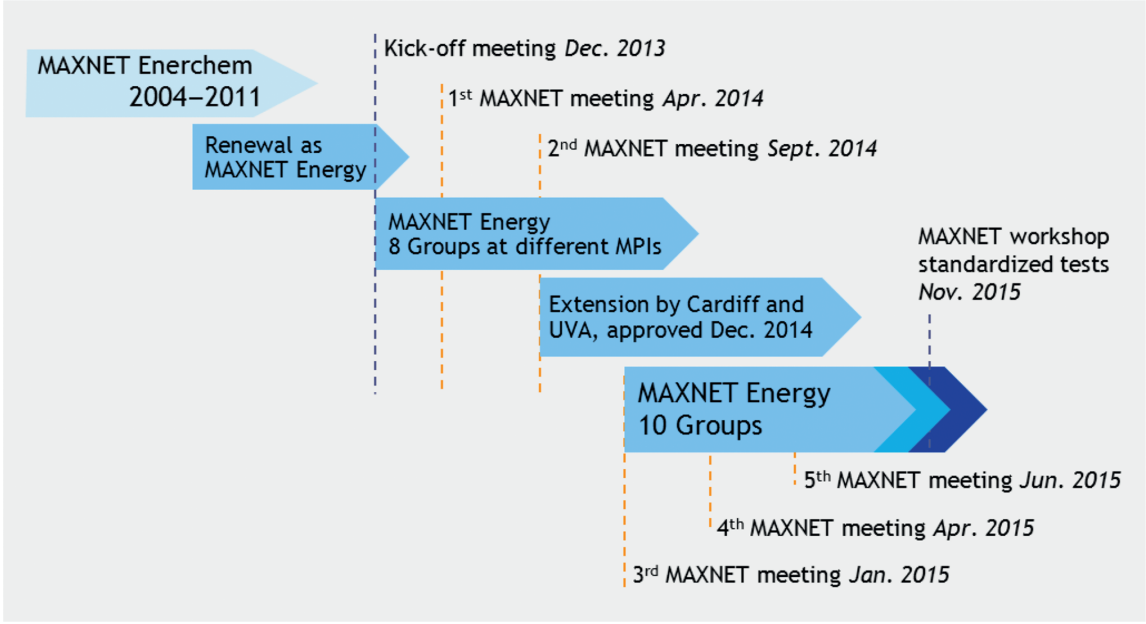

Figure 2: Timeline of the MAXNET Energy initiative of the Max Planck Society.

spectrum of materials science, molecular catalysis, system analysis and engineering solutions.

Overall, the MAXNET Energy consortium includes 10 participating groups, over 35 scientists and an annual budget of EUR 1 million. The span of the projects within MAXNET Energy ranges from atomistic insight of catalytic reactions to details of engineering solutions for technical systems, from quantum simulation to synchrotron measurements and from synthetic chemistry to materials science (see Figure 3).

As the central subject of research, the MAXNET consortium has decided to choose the most prominent but also most complex problem - besides the hydrogen evolution (HER) and oxygen reduction reaction (ORR), the anodic $\boldsymbol{O E R}$ will be in the focus of the activities of groups in the MAXNET Energy initiative. The following paragraphs give an overview over the different partners and the projects the various institutions are contributing to the portfolio of fundamental research within MAXNET Energy:
MPI CEC, Mülheim a.d. Ruhr - Electrode materials, electrocatalyst simulations

In the department of Molecular Theory and Spectroscopy at the MPI CEC in Mülheim, the work is focused on investigating elementary reactions in electrocatalysis. For this purpose, state-of-the art quantum chemical methods are applied in order to assess reactions paths or to interpret spectroscopic data. Here, reactions like the oxygen reduction/evolution and catalyst degradation mechanisms are of special interest. In the department for heterogeneous reactions several catalysis and electrocatalysis related aspects are investigated ranging from novel carbon materials as supports or catalysts (see Section 2.2.3) to fundamental spectro-electrochemical investigations for the OER on established catalysts like iridium or novel materials like manganese (see Section 2.2.2).

MPIE, Düsseldorf - Electrocatalysts and catalyst materials The Interface Chemistry and Surface Engineering department at the MPIE has a long standing tradition
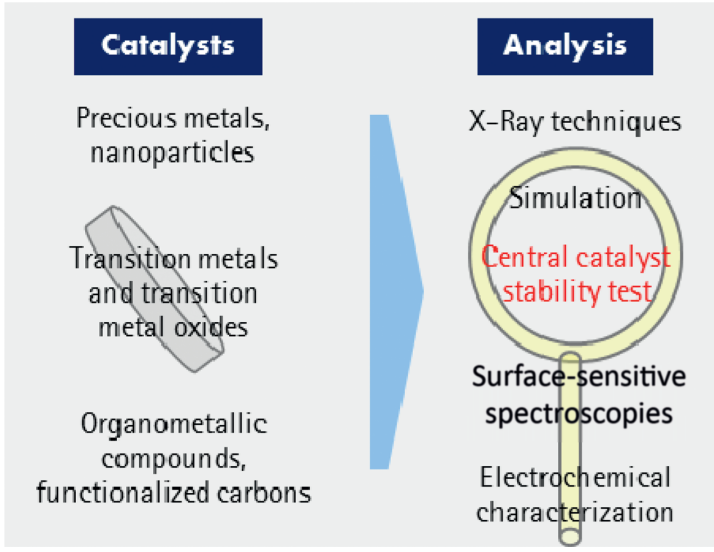

\section{Components}

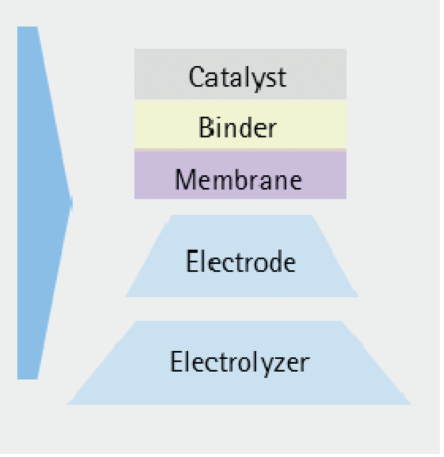

Figure 3: The full bandwidth of expertise within MAXNET Energy and its range from catalysts and material design to advanced analysis techniques up to synthesis, study and design of electrolyser components. 
in research on electrochemical reactions and physical properties of surfaces and interfaces, in particular related to stability of materials, corrosion processes and corrosion protection. The focus of the department within MAXNET Energy is on the fundamental understanding of the decisive surface processes during oxygen reduction/evolution, metal dissolution and de-alloying, predominantly utilizing in-situ surface sensitive investigation techniques as a basis (see Section 2.2.1).

\section{MPIP, Mainz - Molecular catalysts}

The group of synthetic chemistry at the MPIP focuses on the development of precious metal-free, well-defined molecular catalysts as well as carbon-based solid-state materials. The major aim of the work is to control the structures of the molecules and carbons at the atomic level to achieve high performance and to fundamentally understand the correlation between the structures of electrocatalysts and their performance.

\section{MPI DKTS, Magdeburg - System analysis of water} electrolysis

In the department of Process Systems Engineering at the MPI DKTS in Magdeburg, the main focus is on the system analysis of water electrolysis. Optimal design of technical electrodes based on novel catalyst materials supplied by project partners is one of the aspects which have to be explored within the system framework. In this respect, electrochemical characterizations in technical electrolysers and specially designed halfcells are closely combined with mathematical modeling of energy, mass and charge transport processes (see Section 2.3).

\section{MPIKG, Potsdam-Golm - Catalysts/Electrode materials} The Colloid Chemistry department at the MPIKG focuses on the synthesis and application of structure-function controlled inorganic and organic materials. Through precise control of all synthesis steps we aim to prepare materials with tailor-made properties, here for (photo) catalysis and energy storage: Porous carbons with defined doping-patterns show superior performance in the ORR, a system solely based on carbon and nickel possesses unexpected, outstanding properties in the OER, and special polymers are promising as alternative binders in the final devices (see Section 2.1.2).

MPI KoFo, Mülheim a.d. Ruhr - Electrocatalyst materials The focus of Max-Planck-Institut für Kohlenforschung within the MAXNET Energy consortium is design of well-defined nanostructured materials by using the hard templating approach for electrochemical water oxidation. The main emphasis is on composite binary and ternary transition metal oxides to develop more effective oxygen evolution catalysts based on non-precious metals (see Section 2.1.1).

\section{FHI, Berlin - Electrocatalysis}

The electrochemistry research group, hosted by the Department of Inorganic Chemistry of the Fritz Haber Institute, is composed of a multidisciplinary team of scientists performing experimental and fundamental research on a vast portfolio of solid state materials. The principal aim of the electrochemistry group is to study the fundamental physicochemical processes that are involved in energy storage systems for storing intermittent renewable energy.

MPI CPFS, Dresden - Novel intermetallic compounds for electrocatalysis

The main aim of work at the MPI CPFS within the MAXNET Energy consortium is the development of novel catalysts for electrochemical processes, e. g. water splitting reaction. In the large variety of possible catalysts, intermetallic compounds are one of the promising groups of materials. These exhibit a huge width of possible composition, crystal and electronic structure. Investigations of chemical (catalytic) properties are in focus of the research area Chemical Metal Science of the MPI CPfS. Fundamental understanding of the relationship between their structure and (electro)chemical properties offers the opportunity to design and synthesize new catalysts with improved performance.

Cardiff Catalysis Institute, Cardiff - Nanoparticles in catalysis

The Cardiff Catalysis Institute, based at Cardiff University, carries out research focused on understanding the relationship between nanoparticle properties and catalytic activity. Researchers there work toward understanding the formation of nanoparticles, including gold-palladium alloys along with other bimetallic and trimetallic systems, and how to control the morphology and size of nanoparticles through tailored preparation methods. These catalysts are utilized for a variety of reactions including hydrocarbon activation, hydrogen peroxide synthesis, biomass conversions and photocatalytic processes.

University of Virginia, Charlottesville - Molecular and heterogeneous, analysis and engineering solutions in catalysis 
Researchers at the University of Virginia in Charlottesville who are partnering with the Max Planck Society on new energy processes span the Departments of Chemical Engineering, Chemistry and Materials Science and Engineering, and Mechanical and Aerospace Engineering. Research projects under MAXNET Energy include new materials for electrocatalytic conversion of small molecules (e. g., carbon dioxide reduction), new catalysts for the transformation of light alkanes from natural gas to liquid fuels, nanostructured electrodes for photoelectrochemical water oxidation, and the development of new concepts for capture and utilization in materials processing of high value heat from solar energy systems which is traditionally lost as waste heat. A particular focus is collaborative efforts at the interface of homogeneous and heterogeneous catalysis.

\subsection{MAXNET energy - cooperation coordinated}

In science, finding a balance between complete freedom in action and rigorous focus of research to solve a given problem has always been the subject of debate. Two extreme opinions would be: If science is understood as breeding ground for novel ideas that might eventually initiate a technology push process, the unfolding projects must not be steered or directed. If solving problems that originate from a market pull is the aim, development must require focused, directed problem-solving strategies in order to find the best solution. Within MAXNET Energy, a balance is aimed at, which - due to its focus on fundamental research - lies on innovation in materials and in-depth understanding by analysis. Certainly, Max Planck's famous quotation that "insight must precede application" also stands as the guiding principle in this work. However, in order to focus and optimally connect the different activities, MAXNET Energy is designed in a unique way, including two dedicated modes of cooperation that go beyond bilateral interaction or common-aim research compounds. These can be characterized as "shared" and "joined" mode of cooperation, in combination aimed at facilitating focused development and maximal freedom in research at the same time.

The shared mode includes cooperation by sharing expertise, samples and materials or resources. This way, the knowledge and facilities within MAXNET Energy are used in an optimal way and communication and cooperation are facilitated. This is supported by a lively exchange of personnel at the working level, such that postdocs and PhDs working in one group can acquire expertise of other groups with the network.

In the joined mode, participants define and use common reference systems, standards and standard protocols for materials, systems and measurements. As a consequence, each working group uses predefined common experimental parameters in order to guarantee the same experimental conditions and thus comparability and transferability of the results between all groups. A central point is the catalyst stability testing unit, which offers its service to all participants and is supposed to guide catalyst development toward systems that are not only active but also stable (see Section 2.2.2).

This way, added value for each participant is generated not only by the availability of state-of-the-art catalysts and materials, advanced analysis methods or novel upscaling approaches but also by allowing each group to gain an insight into the special problems that arise at different levels of complexity in synthesis, analysis and engineering. For instance, materials scientists benefit from interaction with engineers building electrolyser cells, chemists working on novel catalysts benefit from profound experience in electrochemical analysis and theoreticians benefit from experimentalists insight. As both interaction schemes are combined at the different levels of cooperation, the wide variety of materials developed in the consortium can be compared on common ground, unified test protocols ensure that globally comparable results are obtained in the analytics and clear specifications allow for target-driven development, once a novel system has been explored (see Figure 3).

\section{Overview of selected results and topics}

In the following, some research highlights of work within MAXNET Energy will be outlined. The three pillars of scientific expertise - catalysts and materials, analysis and testing, devices and components (see Figure 3) - are represented by examples from various groups within the consortium.

\subsection{Novel catalysts synthesis}

The efficiency of overall water splitting is limited by the slow kinetics of the water oxidation half reaction. Promising 
performances have already been realized; however, the state-of-the-art electrodes are based on precious metals such as platinum (HER) or $\mathrm{IrO}_{2} / \mathrm{RuO}_{2}$ (OER), where high costs as well as (fuel) cross-over effects render them unfeasible in the long term and especially not on a larger scale $[5,6]$. The entire "water splitting process chain" therefore calls for sustainable, cheap and readily available alternative electrode materials $[7,8]$.

\subsubsection{Transition metal oxides}

Due to their flexible and precise synthetic conditions, ordered mesoporous materials provide an excellent platform for the evolution of the key parameters in a catalytic process [9]. In the group of heterogeneous catalysis at the MPI KoFo, we have been focusing on design of well-defined mesostructured materials to evaluate the importance of various physical and chemical parameters on the efficiency of the electrocatalyst for water oxidation. Ordered mesoporous cobalt-based-oxides have shown outstanding activity and stability due to their high surface area/porosity and rigid framework. Figure 4 shows the typical overview of cubically ordered $\mathrm{Co}_{3} \mathrm{O}_{4}$ that was nanocast from cubicordered mesoporous silica (KIT-6) and the comparison with $\mathrm{Co}_{3} \mathrm{O}_{4}$ nanoparticles $(\sim 6 \mathrm{~nm})$ for water oxidation reaction. By varying the aging temperature of silica, the porous structure and eventually, the surface area of $\mathrm{Co}_{3} \mathrm{O}_{4}$ replica can be straightforwardly tuned. It was found that the increase in surface area positively affects the catalytic activity [10]. Surface modification of $\mathrm{Co}_{3} \mathrm{O}_{4}$ with various transition metals has been conducted and cobalt copper mixed oxides show superior activity than pristine $\mathrm{Co}_{3} \mathrm{O}_{4}$ [11]. The effect of iron doping in $\mathrm{Co}_{3} \mathrm{O}_{4}$ was also investigated and it was shown that small amount of $\mathrm{Fe}$ (Fe/Co 1/64) could tune the symmetry of the replica and improve the activity [12].
Furthermore, the influence of loading amounts on the structure evolution and catalytic activity of $\mathrm{Co}_{3} \mathrm{O}_{4}$ is studied systematically based on the nanocasting process from hexagonally and cubically ordered mesoporous silica [13]. So far, we have shown that the catalyst composition, crystal structure, morphology, geometry, symmetry, and the textural parameters of the materials greatly influence their performances. The ongoing research focus is design of binary and ternary oxides to boost activity of cobalt-based oxides toward the water oxidation.

\subsubsection{Metal carbon composites, nitrides, metal-free carbon and carbon nitride coatings}

As all processes are connected, it is self-evident that the respective materials possess similar characteristics and can sometimes even be the same, i. e. potentially bifunctional catalysts represent the system of choice also from a simplistic, practical point of view. In recent years, the Max Planck Institute of Colloids and Interfaces (MPI KG) has contributed to this field with fundamental studies on the synthesis and investigation of different material classes. Here, metal carbon composites, metal nitrides and pure carbons are highly interesting as they are not only rather cheap but also scalable. In composites, the carbon contributes favorably in terms of electrical conductivity, stability, porosity and heterojunction formation while metal nitrides possess superior electronic tunable properties. First examples of carbon-only materials were also shown to be active catalysts suggesting the possibility of future metal-free systems.

\subsubsection{Metal carbon composites}

We recently found different ways for the very efficient and straightforward synthesis of metal carbon composites. One
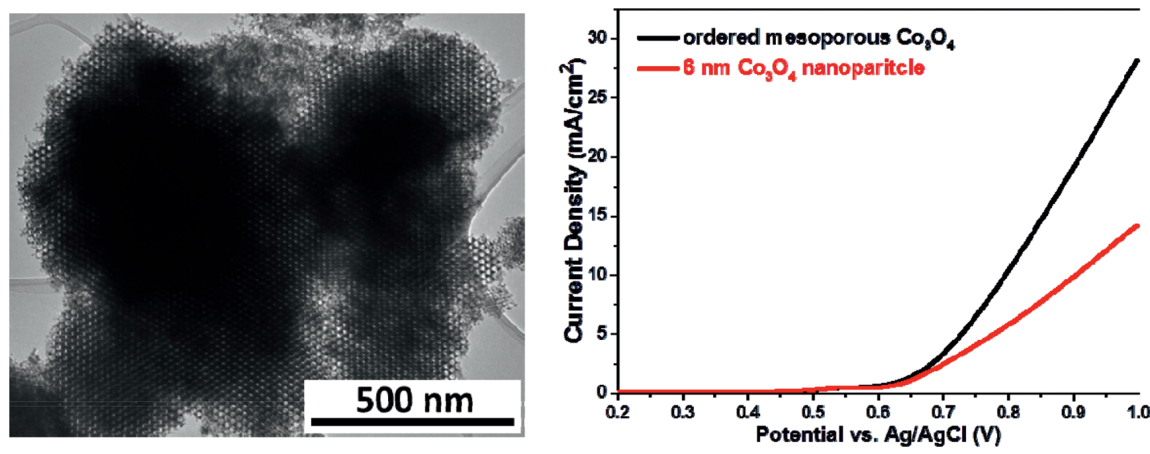

Figure 4: Left: Typical TEM image of cubically ordered mesoporous $\mathrm{Co}_{3} \mathrm{O}_{4}$. Right: Comparison of ordered mesoporous $\mathrm{Co}_{3} \mathrm{O}_{4}$ with $\mathrm{Co}_{3} \mathrm{O}_{4}$ nanoparticle $(6 \mathrm{~nm})$ for electrochemical water oxidation. Catalysts $\left(\sim 0.12 \mathrm{mg} / \mathrm{cm}^{2}\right)$ were dispersed on glassy carbon electrode and the experiments were conducted in $0.1 \mathrm{M} \mathrm{KOH}$ electrolyte. 
example is the filter-paper-based approach with $\mathrm{Ni} / \mathrm{NiO}(\mathrm{OH})$ particles@ nitrogen-doped carbon where the cellulose serves as both carbon source and support. Infiltration with nickel acetate and phenanthroline as ligand and nitrogen-source results in highly active bifunctional catalysts for OER and HER after thermal treatment with low onset potential (330 mV vs. RHE), high current density ( $j>25 \mathrm{~mA} \mathrm{~cm}^{-2}$ at $\eta=430 \mathrm{mV}$ ), good kinetics (Tafel slope of $44 \mathrm{mV} \mathrm{dec}^{-1}$ ), and a very favorable stability $(<5 \%$ decay after $10 \mathrm{~h}$ electrolysis) [14].

Another approach includes the application of commercial blood powder, which is a cheap product from the animal feed industry, containing all necessary ingredients such as carbon, nitrogen, sulfur, and Fe-N coordination sides (from the Heme complex, see Scheme 1) suspected to generate catalytically highly active sites. In combination with $\mathrm{CaCO}_{3}$ as a hard template/activation agent followed by $\mathrm{Co}_{3} \mathrm{O}_{4}$ nanoparticle decoration, sponge-like $\mathrm{Co}_{3} \mathrm{O}_{4} @$ nitrogen-doped carbon composites were obtained with promising catalytic activities in both OER and the reverse ORR in alkaline conditions [15]. The catalytically active sites seem to be different cobalt-nitrogen or cobalt-phosphorous coordination sites making it comparable to commercial platinum/carbon ORR and iridium oxide OER catalysts (half-wave potential of 0.83 $\mathrm{V}$ vs. RHE at the ORR side and a characteristic overpotential of only $380 \mathrm{mV}$ at $10 \mathrm{~mA} \mathrm{~cm} \mathrm{~cm}^{-2}$ at the OER side). Ultimately, this composite is also a promising bifunctional alternative for reversible fuel cells/electrolysers and metal-air batteries.

\subsubsection{Nitrides}

In comparison to the original metal, the formation of the corresponding nitrides (and phosphides) significantly enhances the materials' properties [16]. Here the nitrogen- metal-bonds alter the electronic properties such as nobility but also nucleophilicity and can result in increased electron density at Fermi level. Especially for the HER, metal nitrides are highly attractive as they possess high binding energy for hydrogen, while deposition of $\mathrm{Ni}(\mathrm{OH})_{2}$ on $\mathrm{Ni}$ leads to facilitated water dissociation by $\mathrm{Ni}(\mathrm{OH})_{2}$ and therefore a higher $\mathrm{H}_{2}$ adsorption on Ni.

Recently, our department reported on the solid state synthesis of nickel-nitride-coated nickel foams ( $\mathrm{Ni}_{3} \mathrm{~N} @ \mathrm{Ni}$-foam) [17]. First, supramolecular complexes of cyanuric acid-melamine with minor amounts of barbituric acid (CMB) are formed on the foam surface, which are converted into $\mathrm{N}$-doped carbon. The latter is then removed via electrochemical cycling leading to nickel nitride at nickel composites, which are very stable under alkaline conditions. These materials furthermore possess high surface areas, a fivefold increase of the electrochemically active surface area and can be obtained in high quantities. Besides extremely low over-potentials, high current densities and good stability for HER, the composite foam also possesses high activities in the OER and the ORR in comparison to the original Ni-foam (see Figure 5).

\subsubsection{Metal-free carbons}

Not only for practical reasons but also for the sake of scientific argument, metal-free catalytically active systems are highly important.

In terms of energy density, batteries reveal the best performance in pure electrochemical storage, and new generations and devices such as metal-air batteries are promising candidates [18]. Here, the gaseous oxygen is reduced at the cathode (ORR) through deloading, whereas through

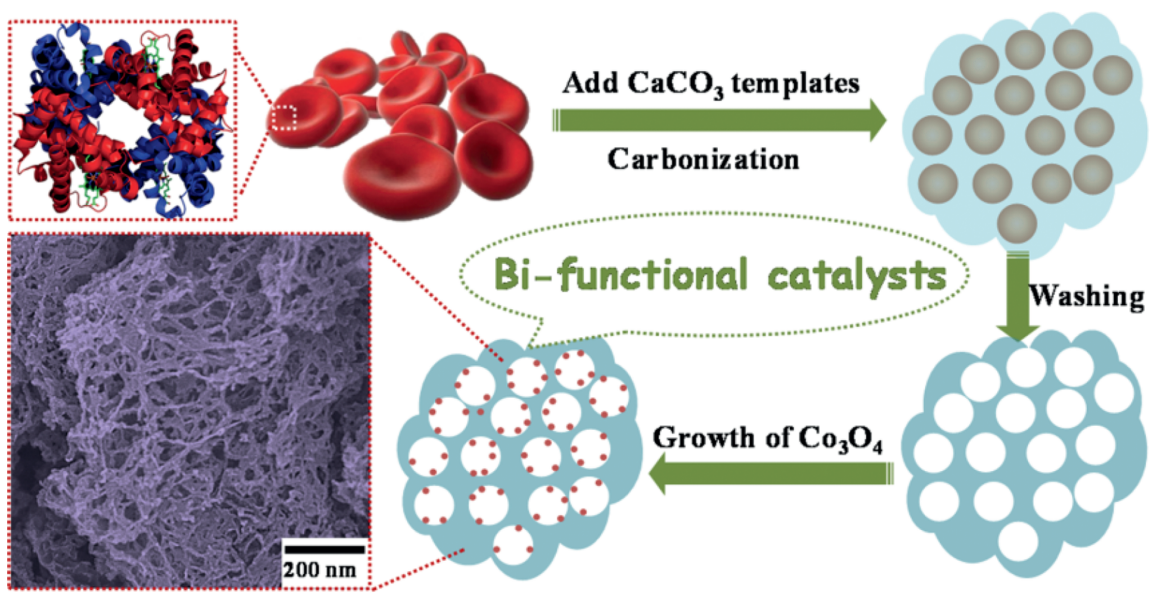

Scheme 1: Schematic of the fabrication process for $\mathrm{Co}_{3} \mathrm{O}_{4}$ decorated blood powder derived heteroatom doped porous carbon (BDHC). Taken from Ref. [15]. 

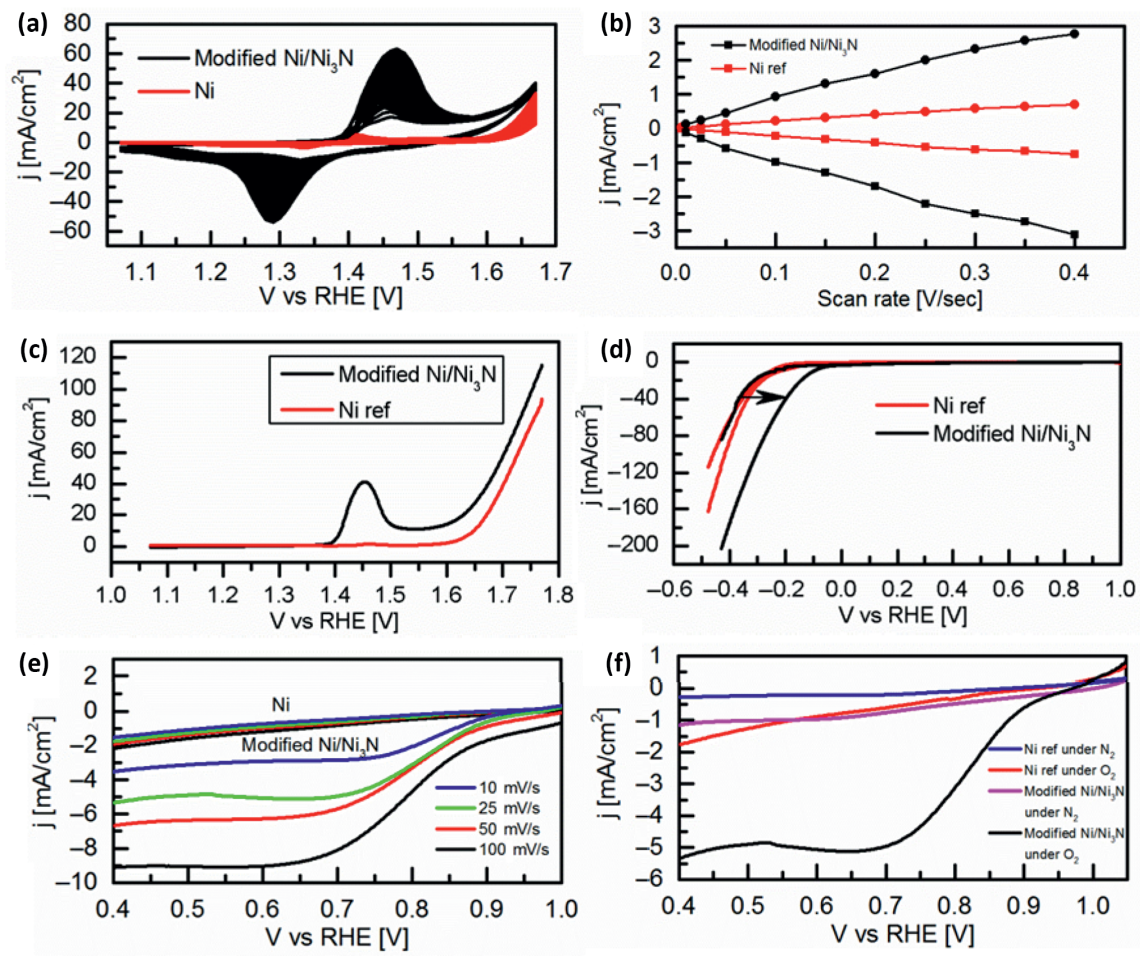

Figure 5: Cyclic voltammetry measurements ( $25 \mathrm{mV} \mathrm{s}^{-1}$ scan rate) (a) The cathodic and anodic charging currents measured at $-0.15 \mathrm{~V}$ vs SCE plotted as a function of scan rate. (b) Linear sweep voltammetry (LSV, $1 \mathrm{mV} \mathrm{s}^{-1}$ scan rate) for the OER (c) and hydrogen evolution reaction of the $\mathrm{Ni} / \mathrm{Ni3N}$-foam compared to the original $\mathrm{Ni}$-foam (d). Oxygen reduction reaction of the foams in different scan rates (e) and the comparison between the foams in nitrogen and oxygen atmosphere using $25 \mathrm{mV} \mathrm{s}^{-1}$ scan rate. All the measurements were performed with 1 M KOH solution. Taken from Ref. [17].

loading the OER produces oxygen from metal peroxides, i. e., oxygen is cycled in the reversible formation of metal oxides and peroxide [19]. This implies several properties, which the electrodes have to fulfill and N-doped carbon materials are currently under investigation as a replacement for noble metals.

We recently demonstrated that ionic liquid-derived carbons, which were obtained from hard templating using silica nanospheres, are promising candidates as they possess high chemical stability, tunable pore structures and high conductivities, i. e. they can be denoted as "noble carbons" [20]. Further improvement is expected via the possibility of targeted modification on the molecular as well as morphological level.

Benefiting from years of expertise in heteroatommodified carbons, we were recently able to present a method for morphology and porosity control of ionic liquid-derived carbons using salts as a porogen [21]. Here, salts offer homogeneous reaction media and eventually allow for more efficient cross-linking while demixing and percolation structures form nanoporous, high surface area nitrogen-doped carbons. These aerogel-like functional carbons revealed very favorable ORR activities without the use of any metal, i. e. diffusion-limited currents of up to $6.1 \mathrm{~mA} \mathrm{~cm}{ }^{-2}$ in acidic media could be generated even better than commercial Pt-catalysts [22]. In this regard, very high salt concentrations were chosen, leading to particulate monolithic materials with remarkable performance in basic as well as acidic conditions. This is attributed to an optimized pore transport system and a high number of active and accessible catalytic sites while sustaining a high level of electrical conductivity and chemical stability.

\subsubsection{Carbon nitride coatings}

Besides $\mathrm{N}$-doped carbons, a further alternative for noble metal-based systems is carbon nitrides $\left(\mathrm{C}_{3} \mathrm{~N}_{4}\right)$. In the last years, this material class was shown to be an efficient catalyst for various reactions [23]. However, so far, it was only possible to generate powders, which complicate the handling especially in regard to device fabrication. Just recently, we found a method to coat conducting surfaces, which is based on the supramolecular assembly of precursors guaranteeing intimate contact between carbon nitride (semi-conductor) and the conductive substrate [24]. This offered the possibility to prepare efficient 
heterogeneous organocatalysts for HER, based on hydrogen-bonded cyanuric acid melamine (CM) supramolecular complex coated on different substrates, being one of the first metal-free systems for this purpose. In general, higher activities were found for basic conditions and we believe that this is due to the high surface amine termination of the carbon nitride rods, which increase the hydrogen concentration on the surface $\left(\mathrm{H}_{\mathrm{ads}}\right)$. Additionally, positively charged carbons in vicinity to the electron-withdrawing nitrogen atoms can easily adsorb $\mathrm{OH}^{-}$groups and $\mathrm{H}_{\mathrm{ads}}$ can eventually be recombined to $\mathrm{H}_{2}$. This non-metal HER electrocatalysts possess low overpotential and good current densities, which can be further improved by targeted modifications of the substrates and the supramolecular carbon nitrides.

\subsection{Analysis: the importance of catalyst stability studies and common standards}

Compared to the well-studied activity of catalysts for oxygen evolution (OER), the topic of the catalyst durability is much less explored [25]. Typically, in scientific works catalyst stability is only addressed briefly, if at all, despite its significance for the operation of electrocatalytic reactors over extended times. Even a consistent methodology for the evaluation of the stability of such catalysts has not yet been suggested or applied, rendering the comparison of the broad variety of novel catalyst systems complicated to say the least.

Ideally, both parameters - activity and stability should be investigated simultaneously under relevant conditions. Moreover, as experiment is still a prerequisite in new catalysts discovery [26], finding active and stable OER catalysts relies on analytic approaches that are capable of handling the diverse materials available and the different operational parameters. In the following sections ongoing research on catalyst degradation and stability analysis are outlined, including the central stability test applied within MAXNET Energy.

\subsubsection{Fast screening of noble metal based OER catalyst activity and stability with SFC-ICP-MS}

A concept along the lines mentioned above has been realized based on the automated scanning flow cell (SFC) electrochemical platform coupled to inductively coupled plasma mass spectrometer (ICP-MS), developed and currently heavily utilized in the group of Mayrhofer $[27,28]$. Unlike many traditional flow cells typically requiring tedious and expensive electrodes replacement, the V-shaped cell geometry of the SFC setup, schematically shown in Figure 6(a), allows performance screening in a matter of minutes. Thereby, the downstream analysis of corrosion reactions products, e.g. by ICP-MS, ensures that information on activity is complemented with operando-results on catalyst stability.

Employing the SFC-ICP-MS for time- and potentialresolved analysis, Cherevko et al. discovered that during oxide formation and oxide reduction all studied noble metals corrode in a transient process [31]. In addition, OER was found to be an additional destabilizing factor causing enhanced dissolution [31]. It was suggested that an extent of noble metal dissolution in this potential region is directly correlated to the mechanism of water oxidation. Noble metal (hydro-) oxides (all metals are covered by a film of oxide at potentials of OER) directly participating in the OER by providing oxygen atoms from the oxide itself demonstrate improved kinetics (low Tafel slope), but also higher dissolution. The involvement of
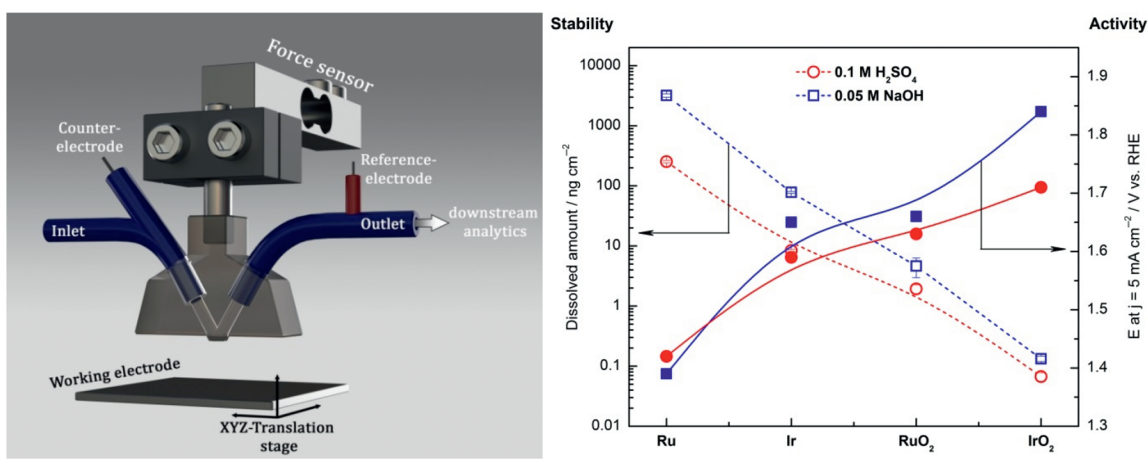

Figure 6: (left) Sketch of the scanning flow cell (SFC). The dimensions of the SFC parts are adjusted for clarity of presentation. Reprinted from Ref. [29] with permission from the Electrochemical Society. (right) Comparison of stability and activity of model thin film $\mathrm{Ru}$, Ir, $\mathrm{RuO}_{2}$, and $\mathrm{IrO}_{2}$ electrodes (based on the data presented in Ref. [30]. 
the oxide in the OER for such metals like $\mathrm{Ru}$ and $\mathrm{Au}$ was experimentally shown using electrochemical mass spectrometry. In contrast, the dissolution of Pt, where the oxide does not participate in the OER, is rather low [32-34]. A thermodynamic explanation for the observed oxide dissolution during OER was recently provided by Binninger etal. [35]. The important outcome of these studies is that in the region of OER potentials, the stability of the material is not only defined by its "nobility," and that there are deviations from the inverse relationship of activity and stability often suggested in literature [36]. As an example, $\mathrm{Au}$ as the noblest of the metals [37] is neither stable during OER in acidic media nor active (at least in the classical view of the activity using exchange current density or onset potential of OER) [38-40].

In acidic media the best known OER catalysts are based on Ru and Ir [41, 42]. Using identical experimental conditions it was recently demonstrated that dissolution of metallic Ir and $\mathrm{Ru}$ (covered with a thin layer of electrochemically formed oxide) is approximately 2-3 orders of magnitude higher than that of thermally prepared $\mathrm{IrO}_{2}$ and $\mathrm{RuO}_{2}$, respectively [30]. A comparison between the two thermally formed oxides shows that the latter is significantly less stable (see Figure 6(b)). None of the electrodes, however, showed a satisfactory durability (in comparison to e. g. Ni) in alkaline media. At high $\mathrm{pH}$ ferrous metals are materials of choice, as they are thermodynamically stable [43]. For studied materials, more stable electrodes were typically less active, confirming at least in this case the earlier mentioned inverse activity-stability correlation [36]. Due to the fact that $\mathrm{Ru}$ is not stable, most of the research on the acidic OER has been devoted to optimization of Ir-based catalysts. Following developments of the low-temperature fuel cells, nanostructuring of electrocatalysts and application of support materials is currently an intensively investigated research topic. Catalyst structure and composition also plays a role in both activity and stability, as was recently shown for Ir@Ti systems [44]. In general, catalysts prepared at higher temperatures were more stable but less active. Once again, however, there are deviations from the activity-stability counterbalance, calling for a more detailed investigation [44]. In a very recent work of Reier et al. [45] the authors suggested that activity and stability of Ir may be related to the coverage of reactive surface hydroxyl groups. The latter can be controlled during synthesis and/or post-annealing.

As most of the presented above examples have aimed for a fundamental understanding of the OER on model systems and to guide new material development, the fast screening capacity of SFC-ICP-MS has not been extensively utilized in combination with high-throughput material synthesis yet. In the future, however, preparation of for instance binary alloy gradient samples using physical vapor deposition techniques [46] or ink-jet printing of nanoparticulated catalysts [47] will ensure a rapid catalyst characterization and optimization.

\subsubsection{Oxygen reduction reaction performance and corrosion evaluation via a combined flow cell - ICP-OES setup}

In contrast to the pioneering, fundamental work of the MPIE group described above [44], which uses a complex and constantly refined flow cell setup, the central stability testing setup is aimed at robust operation and providing service for all groups participating in the MAXNET Energy initiative. As a hub for development, analysis and testing, the unit allows for the broad variety of catalysts to be tested for their activity and - foremost - their stability. Depending on the results of the stability protocol test, further steps in the catalyst development can be initiated. Active but unstable materials can be identified as well as very stable but inactive materials, which can then be investigated for their favorable properties by refined analysis methods. Benchmark for the OER are the typical $\mathrm{IrO}_{2}$ systems, to which novel materials have to be compared on the same ground. This is backed up by a central database in which cross-systems comparisons can help to assess a catalyst potential.

In the approach described in the previous section, an electrochemical flow cell and an ICP-MS are combined into a setup that enables the online stability and corrosion evaluation of OER catalysts. A similar technique is adapted in the central stability testing setup at the MPI CEC in the department for heterogeneous reactions. Here, the performance and corrosion of OER catalysts can be measured simultaneously by matching an electrochemical flow cell with an Inductively Couples Plasma Optical Emission Spectroscope (ICP-OES).

A stability protocol for water splitting applications has been specifically designed to address metal corrosion during various stress test measurements and an optical $\mathrm{O}_{2}$ sensor from Ocean Optics has been employed to measure oxygen evolution before and after the corrosion stress tests to evaluate the catalytic penalty on OER electrocatalysts. It is important to stress the availability of a simultaneous oxygen analysis in order to evaluate the faradaic efficiency of the oxygen evolution in the presence of side reactions like metal dissolution or carbon corrosion.

Our experimental setup, shown in Figure 7, is comprised of an electrochemical flow cell made out of PEEK, a chemical-resistant material, which is specifically chosen to 


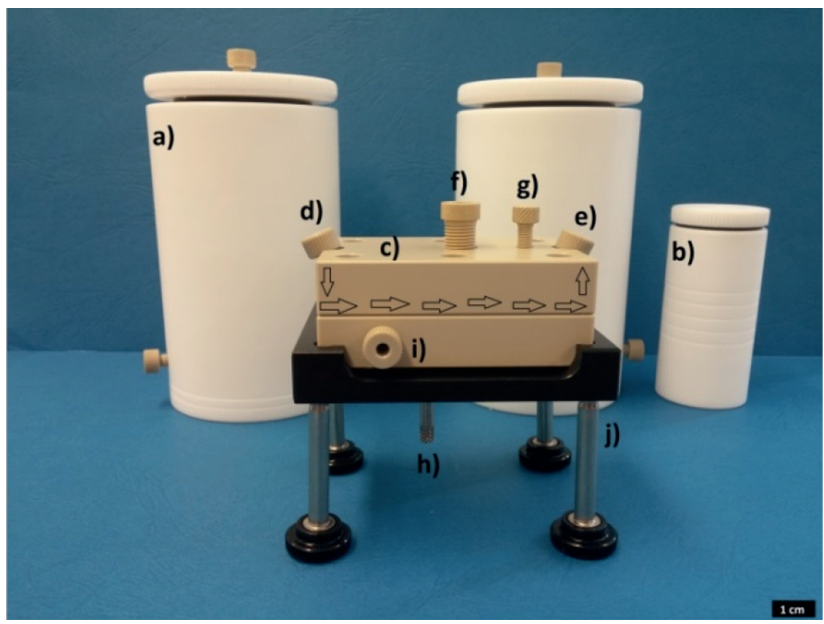

Figure 7: Electrochemical flow cell for performance and corrosion evaluation. (a) Electrolyte reservoirs, (b) reference electrode reservoir, (c) flow cell, (d) inlet, (e) outlet, (f) $\mathrm{O}_{2}$ sensor, (g) Pt-wire counter electrode, (h) working electrode, (i) lugin capillary, (j) flow cell holder.

avoid any oxygen adsorption and permeation through the walls of the cell, which would otherwise cause an $\mathrm{O}_{2}-$ "contamination" and affect the oxygen evolution measurements. The optical oxygen sensor is used to measure the faradaic efficiency of OER catalysts before and after the stability tests. The metal-ion containing electrolyte is fed through the flow cell during the corrosion measurements with the aid of a rotatory-pump, used to introduce liquid samples into the ICP-OES. Our test protocol used for the evaluation of both the performance and the stability of OER catalysts is shown below (Figure 8).

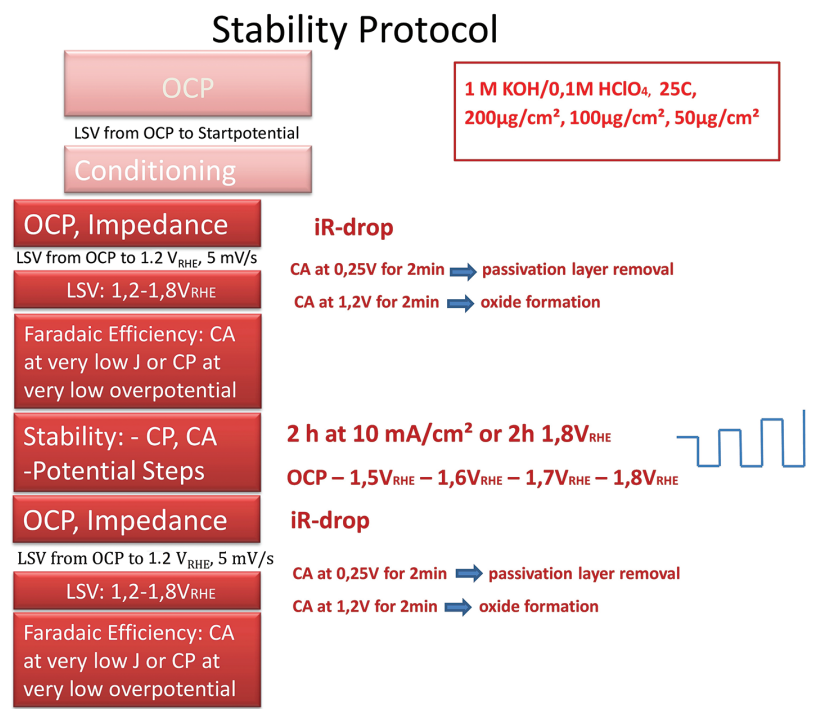

Figure 8: Test protocol for OER catalysts, combines pre-conditioning, performance and corrosion measurements for a rigid OER catalyst evaluation.
All measurements are conducted either in $1 \mathrm{M} \mathrm{KOH}$ or in $0.1 \mathrm{M} \mathrm{HClO}_{4}$ depending on the catalytic material, with internal resistance (IR)-correction. Simple open circuit potential and IR measurements before and after tests can provide us with important information about electrochemically induced catalyst surface changes, while LSV, chronoamperometric (CA) and/or chronopotentiometric measurements provide all the necessary OER performance and stability data for each catalyst tested. Finally, the above data are introduced into a database and used for catalysts stability comparison.

\subsubsection{Focus on the fundamentals of carbon corrosion in carbonanotubes}

As metal-free, cost-effective electrocatalysts currently experience great attention, carbon materials are also considered for the electrode materials in water electrolysis (see above). Carbon is indeed a very attractive material for electrochemical applications as it can exhibit electrical conductivity as well as structural and functional diversity at relatively low cost. Before dimensionally stable metal oxide anodes (DSAs), consisting of Ru or Ir oxides on Ti-based supports had been developed, graphite anodes were commonly used for chlorine and water electrolysis. However, the lifetime of graphite anodes is limited in electrolytic systems due to carbon corrosion.

In the last decades, many different types of carbon structures, such as carbon nanotubes/fibers, graphene, mesoporous carbon, hydrothermal carbon etc. have been developed and intensively investigated. It is generally reported that they show good electrochemical activity and stability. Recently, novel carbon-based catalysts have received attention for the OER [48-52] and, although these materials show surprising OER activity compared to Ir and $\mathrm{Ru}$, their stability for OER is rarely mentioned. In the MAXNET Energy consortium, studying the electrochemical corrosion of novel carbon materials under OER conditions is one of the main objectives. Since carbon is oxidized at high anodic potential, the highly oxidizing OER condition is very harsh for a carbon electrode. Therefore, in the department of heterogeneous reactions at the MPI CEC, we consider the fundamentals of electrochemical degradation of carbon-based catalyst under the environment for OER as a means to address the practical possibility to use carbon as the electrode material in electrolytic processes, either as an electrocatalyst itself or as a support for alternative active species. Moreover, understanding the electrochemical oxidation 

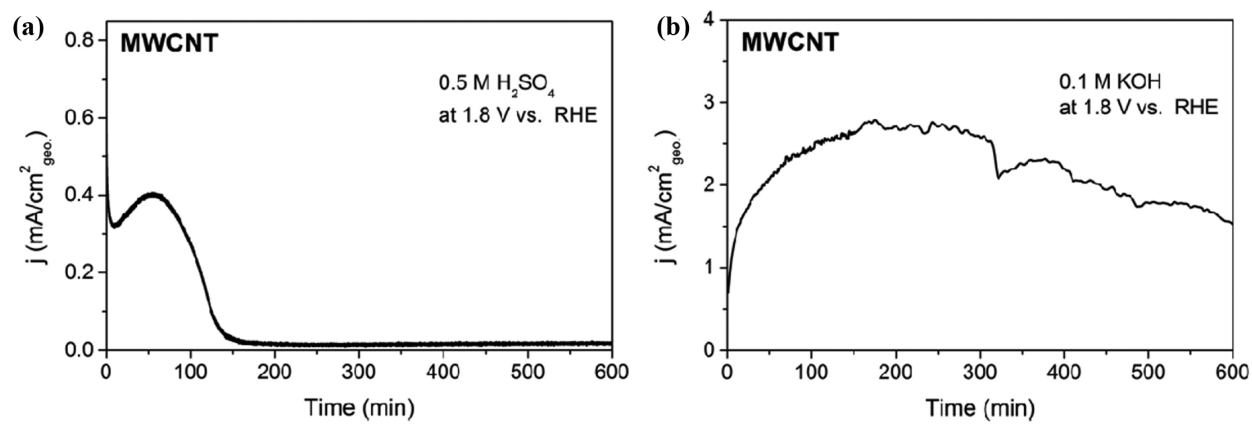

Figure 9: Chronoamperometry of MWCNT at oxygen evolution potential in (a) $0.5 \mathrm{M} \mathrm{H}_{2} \mathrm{SO}_{4}$ and (b) $0.1 \mathrm{M} \mathrm{KOH}$.

of carbon in detail is useful to enhance and influence the application of carbon materials in electrochemical systems.

Figure 9 shows the current-transient curves for multiwall carbon nanotube (MWCNT) at high anodic potential [53]. As oxygen evolution on carbon electrodes is generally accompanied by carbon oxidation, the oxidation current is involved in both reactions of carbon and water oxidation. The initial oxidation current increases due to the formation of a "surface oxide" (oxygen functionalization of the MWCNT), indicating that the carbon surface is activated by the oxidation process. Note that after the initial stage of the current increase, the electrochemical oxidation behavior is different in acidic and alkaline media. In acidic media, the oxides formed on carbon surface slow the reaction kinetics. Thus, it leads to an electrode passivation, resulting in a current value close to zero (Figure 9(a)). On the other hand, the oxidation current in alkaline media initially increases, and then gradually decreases due to carbon degradation. It is inferred from these results that the oxidation current of MWCNT in alkaline media is more pronounced than in acidic media. Therefore, MWCNT might potentially be the base of electrode materials for the OER under alkaline media.

\subsection{From the catalyst to the water electrolysis cell}

Different water electrolysis technologies are currently discussed as options to handle intermittent electricity supply originating from renewable energy sources [54, 55]. Final disclosure (if any) about the "best" technology can be only answered in the system framework where the whole pathway from raw water to pressurized hydrogen, including all pre- and post-processing steps, has to be considered. One of the questions to be answered at the system level is related to the pressure conditions of electrolysis. Depending on the application, hydrogen should be pressurized under different levels (30-200 bars) [56]. Currently, both alkaline and polymer electrolyte (PEWE) electrolysers offer technical solutions for the production of high pressure hydrogen, either by electrolysis at ambient pressure followed by mechanical gas compression (Figure 10, path I) or by high-pressure electrolysis (path II). In addition to these two pathways, a third pathway (path III)) involving electrochemical compression of hydrogen during the electrolysis is thinkable. However, this pathway is technically possible only in combination with PEWE. It has been (a)

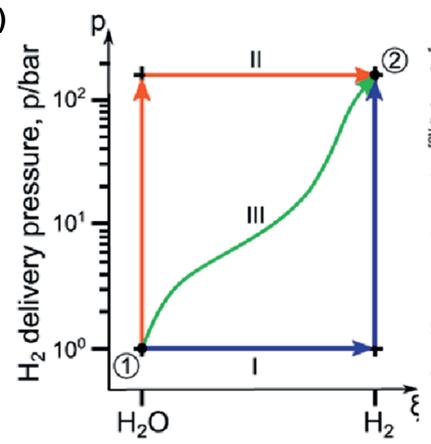

(b)

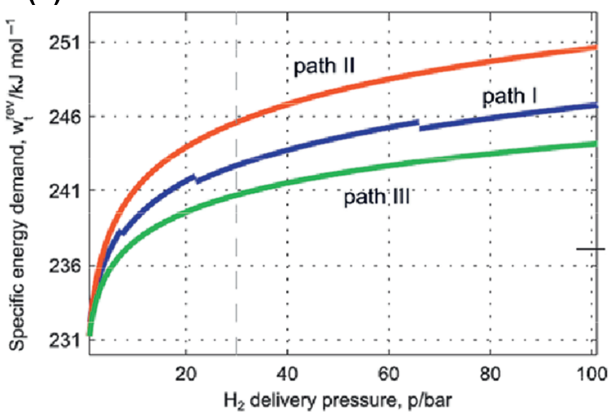

(c)

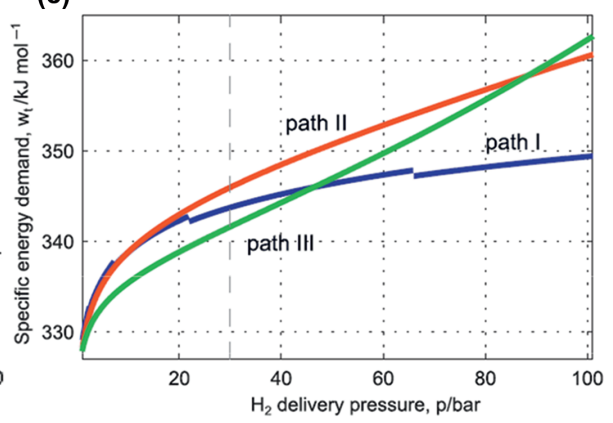

Figure 10: (a) Schematic hydrogen pressure levels for the three PEWE process alternatives. (b) Reversible and (c) irreversible energy demands of the three considered pathways in dependence on the delivery pressure, $\mathrm{T}=333 \mathrm{~K}$ [Taken from Ref. 56]. 
shown (Figure 10) via a detailed energetic evaluation based on a uniform model description of these three hydrogen production pathways that water electrolysis in a cell operating at asymmetric pressure conditions (corresponding to path III) is more favorable at pressures below 40 bars, while at higher pressures electrolysis at ambient pressure followed by mechanical compression (path I) is the better option [56].

But asymmetric pressure levels applied in the anode and cathode compartments of the PEWE cell can lead to significant hydrogen crossover through the polymer electrolyte membrane. In order to ensure safe and efficient operation of the electrolyser, it is important to develop fast and cheap methods to monitor this hydrogen crossover. For this purpose, we have developed a novel in-situ method for the quantification of the hydrogen crossover mass flux [57, 58]. The proposed method features an extremely simple set-up and measurement procedure, as well as high accuracy.

The use of water electrolysers for intermittent storage of renewable energy imposes operation under highly dynamic conditions [56], which may cause accelerated aging of some cell materials. Understanding and controlling the aging mechanisms is imperative for the long-term operation of large-scale electrolyser units. At MPI-DKTS the activity and stability of membrane-electrode-assemblies employing an Ir-based anode catalyst, a Pt-based cathode catalyst and NAFION N117 as polymer electrolyte is currently being evaluated in polymer electrolyte water electrolysis (PEWE) single cell serving as a benchmark for the comparison to novel materials obtained from MAXNET Energy consortium. The stability is tested under a standardized dynamic operating protocol, which mimics PEWE operation as it is foreseen when handling intermittent electricity supply from renewable energy [56]. To test novel materials under technically relevant conditions, they have to be combined to build technical electrodes. This requires addition of different binders and/or supports to the catalyst layers. The final goal is to create welldefined porous catalyst layers with high catalytic activity and low mass transport resistance for water which, at the PEWE anode, is in countercurrent flow to the transport of the gaseous product oxygen. Additionally, catalyst layers should provide sufficiently high ionic and electronic conductivities. All these requirements are difficult to satisfy using current methods for catalyst layer preparation, which often lead to low catalyst utilization, and thus the necessity to use high catalyst loadings which contribute significantly to the electrolyser cost [54]. For this reason, the development of novel strategies for the preparation of tailor-made high-performance catalyst layers has very high practical relevance. A prerequisite for progress in this area is a much better understanding of the molecular interactions between the material constituents of the catalyst layers, which is one of the central goals of the MAXNET Energy consortium.

Acknowledgements: The authors would like to acknowledge the MAXNET Energy research initiative of the Max Planck Society for financial support. Alexander A. Auer would like to acknowledge Marion Tiedtke (MPI CEC) for substantial help with the manuscript and Birgit Deckers (MPI CEC) for help with graphical design.

\section{References}

1. Connolly D, Lund H, Mathiesen BV, Pican E, Leahy M. Renewable Energy 2012;43:47-60.

2. König DH, Freiberg M, Dietrich R-U, Wörner A. Fuel 2015;159:289-97.

3. Zheng Y, Lin L, Wang B, Wang X. Angew Chem Int Ed 2015;54:12868-84.

4. Liu X-M, Lu GQ, Yan Z-F, Beltramini J. Ind Eng Chem Res 2003;42:6518-30.

5. Bing Y, Liu H, Zhang L, Gosh D, Zhang J. Chem Soc Rev 2010;39:2184-202.

6. Duan L, Bozoglian F, Mandal S, Stewart B, Privalov T, Llobet A, et al. Nat Chem 2012;4:418-23.

7. Kudo A, Miseki Y. Chem Soc Rev 2009;38:253-78.

8. Deng X, Tüysüz H. ACS Catal 2014;4:3701.

9. Tüysüz H, Schüth F. Adv Catal 2012;55:127-239.

10. Tüysüz H, Hwang Y, Khan S, Asiri A, Yang P. Nano Res 2013;6:47-54.

11. Grewe T, Deng $X$, Weidenthaler C, Schüth F, Tüysüz H. Chem Mater 2013;25:4926.

12. Grewe T, Deng X, Tüysüz H. Chem Mater 2014;26:3162.

13. Deng X, Schmidt NW, Tüysüz H. Chem Mater 2014;26:6127.

14. Ren J, Antonietti M, Fellinger T-P. Adv Energy Mater 2015;5.

15. Zhang C, Antonietti M, Fellinger T-P. Adv Funct Mater 2014;24:7655-65.

16. Chen W-F, Sasaki K, Ma C, Frenkel Al, Marinkovic N, Muckerman JT, et al. Angew Chem Int Ed 2012;51:6131-5.

17. Shalom M, Ressnig D, Yang X, Clavel G, Fellinger TP, Antonietti M. J Mater Chem A 2015;3:8171-7.

18. Chu S, Majumdar A. Nature 2012;488:294-303.

19. Peng Z, Freunberger SA, Hardwick LJ, Chen Y, Giordani V, Bardé F, et al. Angew Chem Int Ed 2011;50:6351-5.

20. Sakaushi K, Fellinger TP, Antonietti M. Chem Sus Chem 2015;13:1156-60.

21. Fechler N, Fellinger T-P, Antonietti M. Adv Mater 2012;25:75-9.

22. Elumeeva K, Fechler N, Fellinger TP, Antonietti M. Mater Horiz 2014;1:588-94.

23. Wang X, Maeda K, Thomas A, Takanabe K, Xin G, Carlsson JM, et al. Nat Mat 2009;8:76-80.

24. Shalom M, Gimenez S, Schipper F, Herraiz-Cardona I, Bisquert J, Antonietti M. Angew Chem Int Ed 2014;53:3654-8. 
25. Katsounaros I, Cherevko S, Zeradjanin AR, Mayrhofer KJ. Angew Chem Int Ed 2014;53:102-21.

26. Bard AJ. J Am Chem Soc 2010;132:7559-67.

27. Topalov AA, Katsounaros I, Meier JC, Klemm SO, Mayrhofer KJ. Rev Sci Instrum 2011;82:114103.

28. Klemm SO, Topalov AA, Laska CA, Mayrhofer KJ. Electrochem Commun 2011;13:1533-5.

29. Kulyk N, Cherevko S, Auinger M, Laska C, Mayrhofer KJ. J Electrochem Soc 2015;162:H860-6.

30. Cherevko S, Geiger S, Kasian O, Kulyk N, Grote J-P, Savan A, et al. Catal Today 10.1016/j.cattod.2015.1008.1014.

31. Cherevko S, Zeradjanin AR, Topalov AA, Kulyk N, Katsounaros I, Mayrhofer KJ. Chem Cat Chem 2014;6:2219-23.

32. Wohlfahrt-Mehrens M, Heitbaum J. J Electroanal Chem 1987;237:251-60.

33. Willsau J, Wolter O, Heitbaum J. J Electroanal Chem 1985;195:299-306.

34. Diaz-Morales O, Calle-Vallejo F, de Munck C, Koper MT. Chem Sci 2013;4:2334-43.

35. Binninger T, Mohamed R, Waltar K, Fabbri E, Levecque P, Kötz R, et al. Sci Rep 2015;5:12167.

36. Danilovic N, Subbaraman R, Chang K-C, Chang SH, Kang YJ, Snyder J, et al. J Phys Chem Lett 2014;5:2474-8.

37. Hammer B, Norskov JK. Nature 1995;376:238-40.

38. Cherevko S, Zeradjanin AR, Topalov AA, Keeley GP, Mayrhofer KJ. J Electrochem Soc 2014;161:H501-7.

39. Cherevko S, Topalov AA, Zeradjanin AR, Katsounaros I, Mayrhofer KJ. RSC Adv 2013;3:16516-27.

40. Cherevko S, Zeradjanin AR, Keeley GP, Mayrhofer KJ. J Electrochem Soc 2014;161:H822-H830.

41. Fabbri E, Habereder A, Waltar K, Kotz R, Schmidt TJ. Catal Sci Technol 2014;4:3800-21.

42. Zeradjanin AR, Topalov AA, Van Overmeere Q, Cherevko S, Chen X, Ventosa E, et al. RSC Adv 2014;4: 9579-87.

43. Pourbaix M. Atlas of electrochemical equilibria in aqueous solutions. National Association of Corrosion Engineers, 1974, Houston, Texas.

44. Cherevko S, Reier T, Zeradjanin AR, Pawolek Z, Strasser P, Mayrhofer KJ. Electrochem Commun 2014;48:81-5.

45. Reier T, Pawolek Z, Cherevko S, Bruns M, Jones T, Teschner D, et al. J Am Chem Soc 2015 10.1021/jacs.1025b07788.

46. Schuppert AK, Topalov AA, Savan A, Ludwig A, Mayrhofer KJ. Chem Electro Chem 2014;1:358-61.

47. Keeley GP, Cherevko S, Mayrhofer KJ. Chem Electro Chem 2015, Just Accepted, 10.1002/celc.201500425.
48. Walter MG, Warren EL, McKone JR, Boetcher SW, Mi Q, Santori EA, et al. Chem Rev 2010;110:6446-73.

49. Lu X, Yim W-L, Suryanto BH, Zhao C. J Am Chem Soc 2015;137:2901-7.

50. Zhang J, Zhao Z, Xia Z, Dai L. Nat Nanotechnol 2015;10:444-52.

51. Duan J, Chen S, Jaroniec M, Zhang Qiao S. ACS Catal 2015;5:5207.

52. Cheng N, Liu Q, Tian J, Xue Y, Asiri AM, Jiang H, et al. Commun 2015;51:1616-19.

53. Mette K, Bergmann A, Tessonnier J-P, Hävecker M, Yao L, Ressler T, et al. Chem Cat Chem 2012;4:851-62.

54. Yi Y, Tornow J, Willinger E, Willinger MG, Ranjan C, Schlögl R. Chem Electro Chem 2015; In press.

55. Carmo M, Fritz DL, Mergel J, Stolten D. Int J Hydrogen Energy 2013;38:4901-34.

56. Manabea A, Kashiwasea M, Hashimotoa T, Hayashidab T, Katob A, Hiraob K, et al. Electrochim Acta 2013;100:249-56.

57. Bensmann B, Hanke-Rauschenbach R, Pena Arias IK, Sundmacher K. Electrochim Acta 2013;110:570-80.

58. Bensmann B, Hanke-Rauschenbach R, Sundmacher K. Int J Hydrogen Energy 2014;39:49-53.

\section{Bionote}

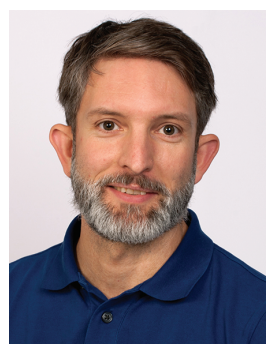

\section{Alexander A. Auer}

Max Planck Institute for Chemical Energy Conversion, Stiftstr 34-36, 45470 Mülheim an der Ruhr, Germany, Alexander.Auer@cec.mpg.de

Alexander A. Auer studied chemistry in Köln and finished his PhD with Prof. J. Gauss in 2002. After a Postdoc with Prof. M. Nooijen in Princeton, USA and Waterloo, CA, he became Junior professor for theoretical chemistry at the TU Chemnitz. In 2009 he was appointed group leader at the MPIE in the department for interface chemistry and surface engineering ( $M$. Stratmann) and is permanent group leader at the MPI CEC in the department of molecular theory and spectroscopy (F. Neese) since 2011. His work focuses on the development and application of electronic structure methods in spectroscopy, catalysis and materials science. 